\title{
Proposal of a Chemical Mechanism for Mini-Beam and Micro-Beam Efficacy
}

\author{
Riccardo Dal Bello ${ }^{1,2,3 \dagger}$, Tobias Becher ${ }^{1,2 \dagger}$, Martina C. Fuss ${ }^{4}$, Michael Krämer ${ }^{4}$ and \\ Joao Seco ${ }^{1,2 *}$
}

${ }^{1}$ Division of Biomedical Physics in Radiation Oncology, German Cancer Research Center (DKFZ), Heidelberg, Germany, ${ }^{2}$ Department of Physics and Astronomy, Heidelberg University, Heidelberg, Germany, ${ }^{3}$ Department of Radiation Oncology, University Hospital Zurich, University of Zurich, Zurich, Switzerland, ${ }^{4}$ Biophysics Department, GSI Helmholtzzentrum für Schwerionenforschung, Darmstadt, Germany

\section{OPEN ACCESS}

Edited by:

Yolanda Prezado,

INSERM U1021 Signalisation normale et pathologique de l'embryon aux thérapies innovantes des cancers,

France

Reviewed by:

Morgane Dos Santos,

Institut de Radioprotection et de Surreté Nucléaire, France

Till Tobias Böhlen,

Center Hospitalier Universitaire

Vaudois (CHUV), Switzerland

Rachel Delorme,

UMR5821 Laboratoire De Physique Subatomique Et Cosmologie (LPSC),

France

*Correspondence: Joao Seco

j.seco@dkfz-heidelberg.de

tThese authors have contributed equally to this work

Specialty section:

This article was submitted to

Medical Physics and Imaging,

a section of the journal

Frontiers in Physics

Received: 22 May 2020 Accepted: 11 September 2020

Published: 22 October 2020

Citation:

Dal Bello R, Becher T, Fuss MC, Krämer M and Seco J (2020) Proposal of a Chemical Mechanism for Mini-Beam and Micro-Beam Efficacy.

Front. Phys. 8:564836.

doi: 10.3389/fphy.2020.564836
This simulation study proposes a chemical mechanism to define a surrogate to the tumor control during micro- and mini-beam radiation therapy (MBRT). The main focus is proton-MBRT (pMBRT) and the methods developed are applied also to photon-MBRT (MRT). In both cases, the classical interpretation of physical dose cannot be used to explain the observed biological effect and a change of paradigm may be required. MBRT was reported to provide tumor control with reduced side effects when compared to standard dose delivery. The underlying mechanisms leading to a differential response of the normal tissue and the tumor are still unknown. In this work, we propose a chemical mechanism to describe the efficacy of MBRT. The model was developed starting from the observation that pMBRT led to long term survival without significant side effects of rats implanted with a high-grade glioma. We distribution of a generic radiation-induced molecule or radical could be a surrogate to describe the biological effect. The specific mechanisms leading to cell damage were outside the scope of this work. The molecules and radicals were selected according to a set of properties: (i) they should be stable to allow diffusion achieving coverage of the dose-valleys, (ii) they should reach a steady state in production versus removal, (iii) they should be a product of water radiolysis, and (iv) they should have oxidizing capacity. A convolution model was developed to assess the property (i) keeping the analysis as general as possible. The tumor coverage was defined widening the interpretation of the ICRU-62 recommendations. The properties (ii) and (iii) were investigated with the TRAX-CHEM software. The property (iv) was used to exclude not relevant chemical species. The results show that hydrogen peroxide fulfills all the requirements. Moreover, the modeling of its temporal and spatial distributions demonstrate that a uniform coverage of the target by this reactive oxygen specie (ROS) can be achieved during the beam-on time. The model was compared and proven to be compatible with three independent photon micro-beam and proton mini-beam animal experiments. We conclude that hydrogen peroxide is a good candidate to describe the mini-beam and micro-beam efficacy. Further experiments are proposed to experimentally benchmark the model and to correlate the hydrogen peroxide concentration to the tumor control probability.

Keywords: mini-beam, micro-beam, TRAX, MBRT, proton therapy, proton mini-beam, hydrogen peroxide, spatially fractionated radiation therapy 


\section{INTRODUCTION}

Technological developments play an important role in the improvement of cancer therapy. Radiation-therapy is, in particular, a rapidly evolving field and it is used as a form of treatment for as many as half of the cancer patients [1]. The refinement of the treatment techniques improved patient care and led to an increase in the survival rate. Long term survivors are the ones who potentially benefit the most from the developments aiming to reduce the side effects of radiation-therapy. The most commonly chosen approach to affect the lesions without inducing side effects is improving the conformality of the dose delivery. A prominent example is the increasing usage of advanced photon delivery techniques such as volumetric modulated arc therapy [2]. Further reduction to the dose delivered to normal tissue may be obtained with beams of charged particles. While electron beams can be employed for superficial tumors, high energy proton, and ion beams allow to escalate the dose to deep located target volumes while reducing the burden to the normal tissue due to their physical and biological characteristics [3]. Further improvements will be investigated at the new generation facilities [4]. In general, the approaches adopted in clinical practice aim to achieve tumor control by delivering a uniform dose to the target volume. In pre-clinical studies several other options have been investigated, which not always base their rationale on uniform dose distributions and conformality. A prominent case is the micro- and mini-beam radiation therapy (MBRT), where a spatial pattern of high-dose beamlets alternates with low-dose valleys. This has been investigated in animal experiments with photon beams (MRT) at synchrotron facilities [5] and recently at dedicated light sources [6]. Also recently, proton beams were deployed as well (pMBRT) [7]. The investigation of MBRT requires a change of paradigm. The reduction of the side effects to the organs at risk are not achieved by reducing their integral dose, but thanks to a differential response of normal and tumor tissue to the radiation delivered with a welldefined spatial fractionation. Two recent studies investigated the tumor response to MRT for mice implanted with squamous carcinoma [6] and to pMBRT for rats implanted with highgrade glioma [8]. In the former, the tumor growth delay was compared between a tumor-bearing control group, mice treated with broad-beam and with micro-beams. Two different dose levels were investigated. In the latter, a long term followup was performed to monitor the side effects and tumor response for normal and tumor-bearing animals. The rats were either part of the control group or were treated with minibeams at one dose level. The animal experiments reported that pMBRT preserved the tumor control. The interpretation of such result is the aim of the current study. Moreover, a previous MRT study performed at a synchrotron facility was also taken into account [9]. In this case, Regnard et al. found that the probability to ablate the implanted intracerebral $9 \mathrm{~L}$ gliosarcoma increased by a factor more than 7 when decreasing the spacing of micro-beams from 200 to $100 \mu \mathrm{m}$. Differences in normal tissue toxicity were also observed, but will not be further investigated in the current study, which will focus on the
MBRT efficacy in tumor tissue. All the previous studies included histological analysis.

MBRT has been investigated at two different spatial scales. In both cases, the setup utilizes arrays of parallel thin radiation planes separated by short distances. In first approximation, along the transverse profile, the radiation can be modeled by a series of equidistant rectangular peaks separated by valleys without direct delivery of the beam. We aimed to keep the study as simple as possible in order to avoid biases in the results due to the choice of free parameters. The profiles can be characterized by their center-to-center distance $c-t$ - $c$, i.e., the spacing between the areas with direct radiation, and the full width half maximum of the radiation peaks $F W H M$. In the case of mini-beams, the $c-t-c$ can be as wide as few millimeters and the FWHM up to the millimeter scale [8]. To achieve such beam profiles, dedicated mechanical collimators are typically designed [10]. The presence of a collimator leads on one hand to a reduction of the dose rate with respect to a broad beam irradiation and on the other hand to scattering of the primary radiation. The former is taken into account in the current study by analyzing the total irradiation time, which if long enough may allow the propagation of radiolysis products in the valleys. The latter leads to the presence of a non-zero dose in the valleys, which is then characterized by the peak to valley dose ratio PVDR. High values are achievable with novel techniques such as magnetic focusing for ion beams [11]. The physical dose delivered in the valleys is therefore small compared to the average dose delivered in the tumor, which leads to a significant part of the tumor volume to be under-dosed. Nonetheless, several animal experiments report delayed tumor growth [6], improved survival fraction [12], and tumor control with cases of tumor eradication [8]. Such effects cannot be explained by the low physical dose delivered to the valleys. Alternative interpretations including cell-signaling cascades have been proposed for the normal tissue sparing [13]. Recently, the correlation between tissue damage and the level of reactive oxygen species (ROS) has been proposed [14]. The related radiolysis products can be nowadays accurately modeled by dedicated simulation codes, such as TRAX-CHEM [15]. This work aims to fill the gap in the interpretation of the tumor control observed in MBRT, combining the previously mentioned results to investigate a chemical mechanism for mini-beam and micro-beam efficacy.

This study consists in three subsequent phases. First, we investigate the spatial distributions of the physical dose and the radiolysis products during MBRT. Then, we analyze and model the spatial and temporal evolution of the molecules and radicals produced by the radiation beam. Finally, the model is used to interpret previous MBRT animal experiments.

\section{MATERIALS AND METHODS}

The spatial distribution of ROS was investigated to develop a model interpreting the MBRT efficacy. The current work was based on simulations and it provides a more in-depth analysis of previously published MBRT animal studies. No animal experiments were conducted within the current study. 
The model was developed starting from the observation that pMBRT led to long term survival without significant side effects of rats implanted with a high-grade glioma [8]. It was then applied to MRT used to irradiate mice with squamous carcinoma [6] and rats with intracerebral 9L gliosarcoma [9]. We selected the three previously cited studies since they provided all the parameters required to compare the simulations to the experimental data. Namely, the complete dosimetric description of the irradiation, the total irradiation time, and the observation of a biological effect. We investigated whether the distribution of a radiation-induced molecule or a radical could be a surrogate to describe the biological effect. The specific mechanisms leading to cell damage were outside the scope of this work. Four properties were defined, to restrict the investigation solely to relevant radiation-induced molecules or radicals. The conditions were:

(i) it should be stable to allow diffusion during beam-on, achieving coverage of the dose-valleys

(ii) it should reach a steady state in production versus removal within few microseconds of beam-on

(iii) it should be a product of water radiolysis

(iv) it should have oxidizing capacity to allow damage of proteins, lipids and DNA.

The choice of the conditions was motivated by the following properties. (i) The relevant distance of diffusion is the one that the molecules and radicals can reach during beam-on. In absence of radiation, the reactive chemical species are rapidly removed by antioxidants [16]. (ii) The steady state is required since the time scale of the biological effects is much longer compared to the one of the physics phenomena that trigger the process. Fast processes that do not reach the steady state, while contributing indirectly to the steady state of other processes, are not expected to be directly correlated to a biological effect. The radiationinduced molecules or radicals that reach the steady state are chosen as a potential surrogate for the biological effect. (iii) The surrogate for the biological effect of MBRT should be directly or indirectly generated by ionizing radiation. Therefore, other processes leading to water dissociation were excluded. (iv) The reactivity of the molecule or radical is required in order to induce a chemical or biological effect.

The study was divided in three subsequent phases. First, the property (i) was assessed keeping the analysis as general as possible. We investigated the diffusion of molecules and radicals from their production in the peaks to the valleys. No constraints were imposed at this stage. The details are presented in section 2.1. The second phase investigated the properties (ii) and (iii) with the software TRAX-CHEM [17]. The simulations were based on the properties of the proton mini-beams reported by [8]. The spatial distributions of twelve different molecules and radicals were tracked over several orders of magnitude of their time evolution. The details are provided in section 2.2. Finally, in the third phase, we compared the characteristic diffusion times of the molecules and radicals with the irradiation times adopted in the previous mini-beam [8] and micro-beam [6] animal studies. The methodology of the comparison is presented in section 2.3.

\subsection{Generalization of the Dose Coverage Concept}

\subsubsection{From Physical Dose to $g R M$ Concentration}

This study investigates the secondary water products produced by radiation, known as radiolysis. We define $g R M$ as the generic Radical or Molecule produced during radiolysis, and that diffuses through the cells after being produced. The international recommendation of ICRU established that the dose values in the PTV should be confined within 95 to $107 \%$ of the prescribed dose [18]. This classical concept of physical dose coverage of the target volume is not respected in MBRT, due to the presence of low-dose valleys (Figure 1). We hypothesize that a coverage within 95 and $107 \%$ is not provided directly by the physical dose, but by a secondary product of the interaction between ionizing radiation and water target. As a matter of fact, the values assumed by the physical dose will not enter directly our model, which will focus on the distribution of the secondary products. The $g R M$ is created at the interaction point between the ionizing radiation and the target and it can be any of the radicals and molecules listed in Table 1. Its concentration at the moment of creation is assumed to be directly proportional to the physical dose. Starting from a point-like physical dose distribution and assuming the condition (i) to be respected, at a given time point, the $g R M$ will have a given spatial distribution. Keeping the analysis as general as possible, we model such distribution with a Gaussian function. In a one dimensional representation, the $g R M$ distribution is parameterized by its amplitude $A_{k}$, standard deviation $\sigma_{k}$ and mean $\mu_{k}$. While $\mu_{k}=0$ for the symmetry properties of the problem, the other parameters evolve in time according to the specific properties of each radical or molecule: $A_{k}:=A_{k}(t)$ and $\sigma_{k}:=\sigma_{k}(t)$. Given a physical dose distribution $D(x)$, the relative concentration of $g R M$ can be calculated with a convolution:

$$
\rho_{g R M}(x)=k \cdot D(x) \circledast \frac{1}{2 \pi \sigma_{k}} e^{-\frac{x^{2}}{2 \sigma_{k}^{2}}}
$$

where $k$ is a normalization factor. In other words, the distribution $D(x)$ is converted into $\rho_{g R M}(x)$ through a kernel of width $\sigma_{k}$. Likewise, the prescribed physical dose can be converted into a mean concentration of $g R M$. It is promptly seen that for a classical uniform $D(x)$ respecting the ICRU recommendation, also $\rho_{g R M}(x)$ is within the 95 to $107 \%$ limits. On the other hand, for MBRT, such condition is not respected by $D(x)$ but it may be respected by $\rho_{g R M}(x)$ depending on the value assumed by $\sigma_{k}$.

\subsubsection{Calculation of $\sigma_{k}$ in Previous PMBRT Studies}

Prezado et al. reported that the pMBRT used in their experiments were characterized by a width of $1.1 \mathrm{~mm}$ at $1 \mathrm{~cm}$ depth, PVDR of approximately 6.25 and $c-t-c=3.2 \mathrm{~mm}$ [8]. We calculated the value of $\sigma_{k}$ required for $\rho_{g R M}(x)$ to be within the 95 to $107 \%$ limits for this experiment. The physical dose distribution was reproduced with a series of identical Gaussian functions. A more direct approach adopting rectangular functions was also investigated (Figure 1). The Equation (1) was applied to the physical dose distribution and the uniformity of $g R M$ was recorded at varying $\sigma_{k}$. The smallest $\sigma_{k}$ producing a $\rho_{g R M}(x)$ distribution respecting the ICRU recommendation was recorded 

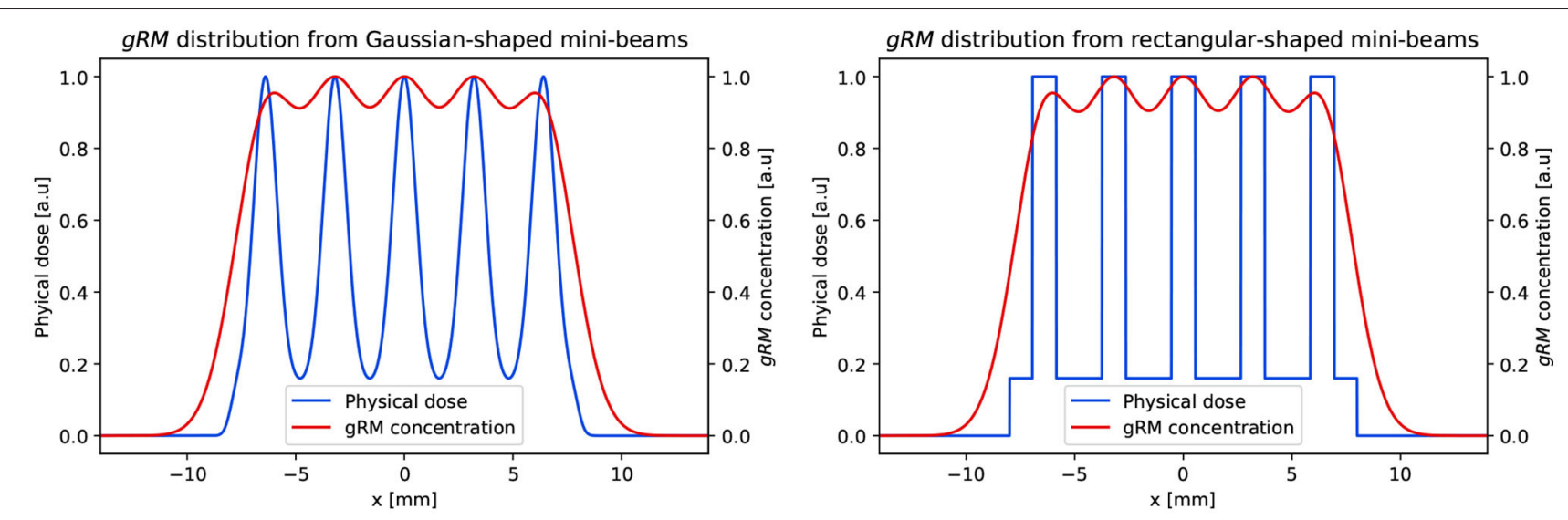

FIGURE 1 | One dimensional representation of the conversion from the physical dose deposited by pMBRT to the spatial distribution of a generic molecule or radical (gRM). Two scenario are compared: (left) Gaussian shaped and (right) rectangular shaped mini-beams. In both cases, the following parameters were adopted:

$c-t-c=3200 \mu \mathrm{m}, F W H M=1100 \mu \mathrm{m}, P V D R=6.25$, and $\sigma_{k}=1250 \mu \mathrm{m}$. The data matches the values in [8], which reported the dosimetric data at a depth of $1 \mathrm{~cm}$.

as $\hat{\sigma}_{k}$. We compared the $\hat{\sigma}_{k}$ values obtained with Gaussian-shaped and rectangular-shaped mini-beams. An exemplary conversion of $D(x)$ into $\rho_{g R M}(x)$ for the two cases is presented in Figure $\mathbf{1}$. The same approach was then applied to MRT experiments. The value of $\hat{\sigma}_{k}$ was calculated for the micro-beam experiment reported by [6]. Here, the dosimetric parameters were: width $=50 \mu \mathrm{m}$ and $c-t-c=350 \mu \mathrm{m}$. The PVDR was not explicitly reported, but the authors say that it was much higher than 48 . We adopted $P V D R=50$ in our simulations. It should be noted that higher values would have negligible influence on the results of the gRM distribution, as its value at high PVDR is dominated by the peak dose. Finally, we calculated $\hat{\sigma}_{k}$ for the two irradiation conditions reported by [9]. In the first case, a $c-t-c=200 \mu \mathrm{m}$ was used and the PVDR was 42.3 at $5 \mathrm{~mm}$. In the second one, a $c-t-c=100 \mu \mathrm{m}$ was used and the PVDR was 14.2 at $5 \mathrm{~mm}$. All the experiments were performed with beams of width $=$ $25 \mu \mathrm{m}$ generated with a dedicated collimator at the European Synchrotron Radiation Facility (ESRF) [19]. We will reference the experiments with the same nomenclature used by Regnard et al., i.e., series $200^{* 1}$ for the first and series 100RL for the second

\subsection{Simulation of the Radiolysis Products \\ 2.2.1. The TRAX-CHEM Software}

The evolution of proton tracks in water through the physical, pre-chemical, and chemical stage was simulated using the TRAX [20] code and its recent extension TRAX-CHEM [17]. Implementation of track chemistry in water [17], the addition of dissolved oxygen [21], and the respective reaction channels have been previously described in detail. In brief, physical particle interactions are simulated with an event-by-event Monte Carlo approach. Ionization and electronic excitation events lead to molecular dissociations whose coordinates and ionization or excitation state are passed over to the prechemical stage together with thermalized secondary electrons. There, molecular

\footnotetext{
${ }^{1}$ The group $200^{*}$ includes the series 200LR1, 200LR2, 200RL
}

dissociation and thermalization of the fragments are simulated and conclude after $\sim 1 \mathrm{ps}$. These radiolytic species are then tracked as they diffuse and react with each other according to well-known rate constants, and the products of reactions amongst themselves or with water or dissolved molecular oxygen are continuously included into the pool of active chemical species, whereas the consumed reactants are removed. The type of radical and coordinates of all active species are recorded at specified intermediate time points. The spatial dissolution of the track structure into a microscopically homogeneous distribution is normally reached at $\sim 1 \mu$ s together with chemical equilibrium. Photon beams were not simulated in the current study. We defined the temporal evolution of the distribution of the molecules based on proton simulations only. This is justified by the fact that the primary radical yields as well as the diffusion process of the molecular species in water are insensitive to radiation quality at low linear energy transfer.

\subsubsection{Temporal Evolution of $\boldsymbol{A}_{\boldsymbol{k}}$ and $\sigma_{\boldsymbol{k}}$}

For radiolysis simulation with TRAX-CHEM, conditions were set as follows. The source was an infinitesimally thin proton beam of kinetic energy $E_{k}=92 \mathrm{MeV}$, i.e., equivalent to the mean energy at $1 \mathrm{~cm}$ depth in water of the beams used in the experiments reported by [8]. It was placed immediately in front of the target volume where interactions can take place. The target was a water cylinder of $3 \mu \mathrm{m}$ height and $10 \mu \mathrm{m}$ radius with an oxygenation value of $6 \%$, resembling physiological conditions at body temperature. Secondary particles (electrons) were followed explicitly through all interaction events until $7 \mathrm{eV}$, i.e., below the threshold for electronic excitations. At that point, the residual kinetic energy was converted into one final displacement in a random direction according to [22]. We tracked over time the distribution of twelve radiolysis products in order to identify a potential $g R M$ fulfilling the requirement (ii). Table 1 summarizes the molecules and radicals tracked. The simulations were sampled at multiple time points from $10^{-12}$ 
to $10^{-5} \mathrm{~s}$. An example of the production and diffusion of $\mathrm{OH}^{\bullet}$, $\mathrm{H}_{2} \mathrm{O}_{2}$, and other species is reported in Figure 2. We first analyzed the temporal evolution of $A_{k}(t)$ for all the molecules and radicals and then $\sigma_{k}(t)$ for the ones respecting the condition (ii).

The total number of molecules or radicals for each of the species listed in Table $\mathbf{1}$ was recorded at multiple time points. In this analysis, the number of events was integrated over the whole target. The species were separated in two categories, depending on their behavior at the latest simulation time point. In the first category, we included the species that did not reach a steady state and were still increasing or decreasing in their total amount. In the second category, we included only the species that reached the steady state, i.e., $\partial A_{k} / \partial t \simeq 0$. The first were excluded from further analysis as the condition (ii) was not respected.

We analyzed the radial positions of the species in the second category. This was done taking into account the absolute distance from the beam axis and collecting the data in histograms. The chosen landmark of the distribution was $P_{68}$, i.e., the 68th percentile. Its value evolved with time. We defined $\sigma_{k}(t):=$ $P_{68}(t)$ and their values were collected at the previously defined time points of the simulation. The temporal evolution of $\sigma_{k}(t)$ was fitted with a power law for $t>t_{1}$, where $t_{1}$ was the first time point of the simulation where $A_{k}(t)$ reached a steady-state. The functional form was chosen in accordance to the data presented in section 3.2. The parameterization was $\sigma_{k}(t)=a \cdot\left(t / t_{0}\right)^{b}$. We fixed the parameter $t_{0}=1 \mathrm{~s}$, which defines the unit for the time.

TABLE 1 | Breakdown of the molecules, radicals, and ions followed in the TRAX-CHEM simulations.

\begin{tabular}{lccc}
\hline$\bullet \mathrm{OH}$ & $\mathrm{H}_{3} \mathrm{O}^{+}$ & $\bullet \mathrm{H}$ & $\mathrm{H}_{2} \mathrm{O}$ \\
\hline$e^{-}$ & $\mathrm{H}_{2}$ & $\mathrm{H}_{2} \mathrm{O}_{2}$ & $\mathrm{OH}^{-}$ \\
\hline $\mathrm{O}_{2}$ & $\bullet \mathrm{HO}_{2}$ & $\bullet \mathrm{O}_{2}^{-}$ & $\mathrm{HO}_{2}^{-}$
\end{tabular}

\subsection{Tumor Coverage by $g R M$ in Previous MBRT Animal Experiments}

Keeping the analysis as general as possible, we assume that a given $g R M$ fulfills the conditions (i)-(iv) and that it can be identified by the methods presented in sections 2.1, 2.2. For such $g R M$, it can be defined a characteristic time $\hat{t}$ at which also the generalized definition of tumor coverage by ICRU is fulfilled. This can be promptly calculated by inverting the parameterization, i.e.,

$$
\hat{t}=t_{0} \cdot a^{-\frac{1}{b}} \cdot\left(\hat{\sigma}_{k}\right)^{\frac{1}{b}} .
$$

This characteristic time can be correlated with the beam-on in the animal experiments, being the latter the only significant time scale for the radiochemical processes. We postulate that (2) corresponds to the minimum beam-on time required for a MBRT irradiation to achieve tumor coverage by $g R M$. This assumption is justified by the phenomena happening at longer and shorter time scales. For beam-on times longer than $\hat{t}$, the steady state of $g R M$ has already been reached and therefore the postulate applies. For beam-on times shorter than $\hat{t}$, the steady state of $g R M$ may not always be reached through simple diffusion at a later time point. Metabolic processes remove molecules and radicals from the target and therefore modify the $g R M$ distribution. Therefore, we directly compared the time scale of $\hat{t}$ with the beam-on time. Given $t_{\text {exp }}$, the beam-on time in a MBRT experiment, the $g R M$ can be a potential surrogate to describe the biological effect if the condition $t_{\exp } \geq \hat{t}$ is met. Therefore, we analyzed previous MBRT animal experiments where tumor control was reported and we compared their $t_{\exp }$ with our calculation of $\hat{\sigma}_{k}$ and $\hat{t}$.

The pMBRT experiment by Prezado et al. was conducted with collimated proton beams [8]. They reported that the irradiation was performed with a dose rate of $\dot{D}=2 \mathrm{~Gy} \cdot \mathrm{min}^{-1}$ at $1 \mathrm{~cm}$ depth. This depth was also the reference point used to report the mini-beam width. The peak dose was $D=70 \mathrm{~Gy}$, which converts into an irradiation time of $\hat{t}=35 \mathrm{~min}=2100 \mathrm{~s}$. This value was used for our analysis.

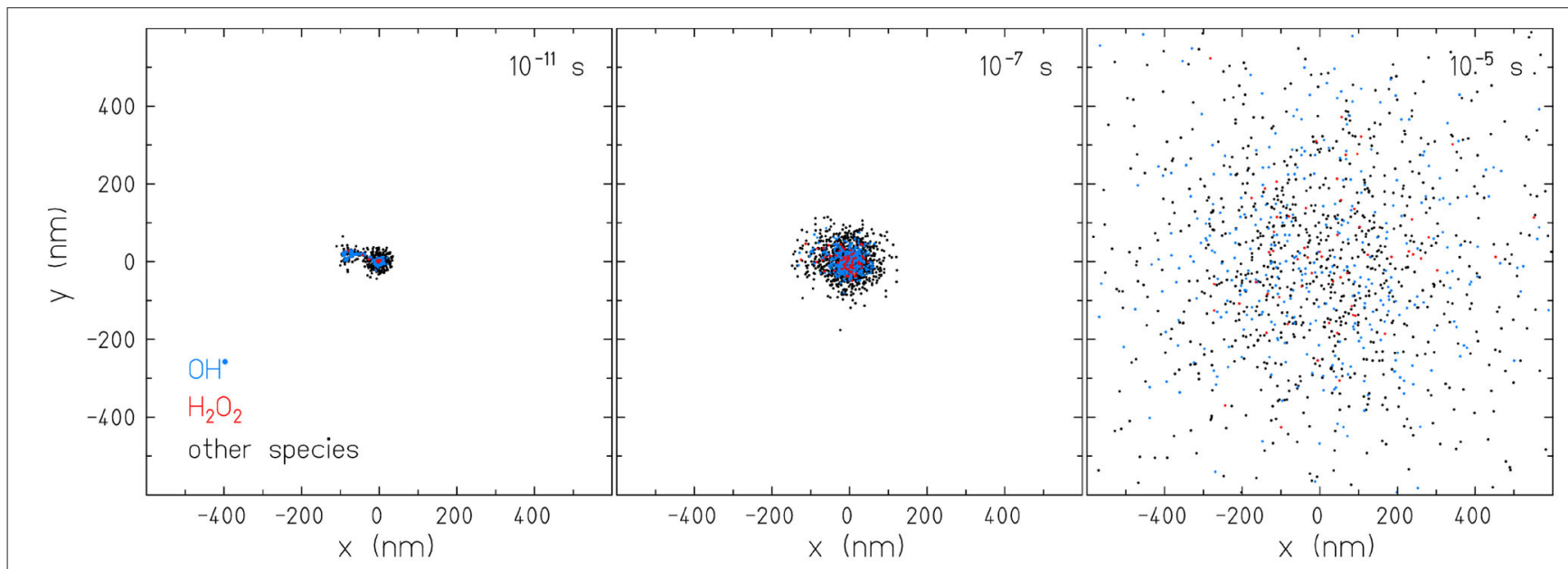

FIGURE 2 | Two dimensional representation of the temporal diffusion of molecules and radicals simulated with the software TRAX-CHEM. The events displayed are produced by a proton beam with kinetic energy $E_{k}=92 \mathrm{MeV}$ in water. 
The MRT experiment by Dombrowsky et al. was conducted with photon beams at the Munich compact light source (MuCLS) [6]. The authors reported explicitly the irradiation time, which varied depending on the experimental setup. The integral dose rate for MRT was up to $0.6 \mathrm{~Gy} \cdot \mathrm{min}^{-1}$. Fluctuations of the instantaneous values of $\dot{D}$ lead to total irradiation times of $(5.6 \pm 0.8) \mathrm{min}$ for the delivery of $D=3 \mathrm{~Gy}$. For the purpose of our model, the shortest irradiation time showing a biological effect is the relevant one. Therefore, we calculated the minimum $t_{\exp }$ that could be achieved with $\dot{D}=$ $0.6 \mathrm{~Gy} \cdot \mathrm{min}^{-1}$, i.e., $t_{\exp }=300 \mathrm{~s}$. This value was used for our analysis.

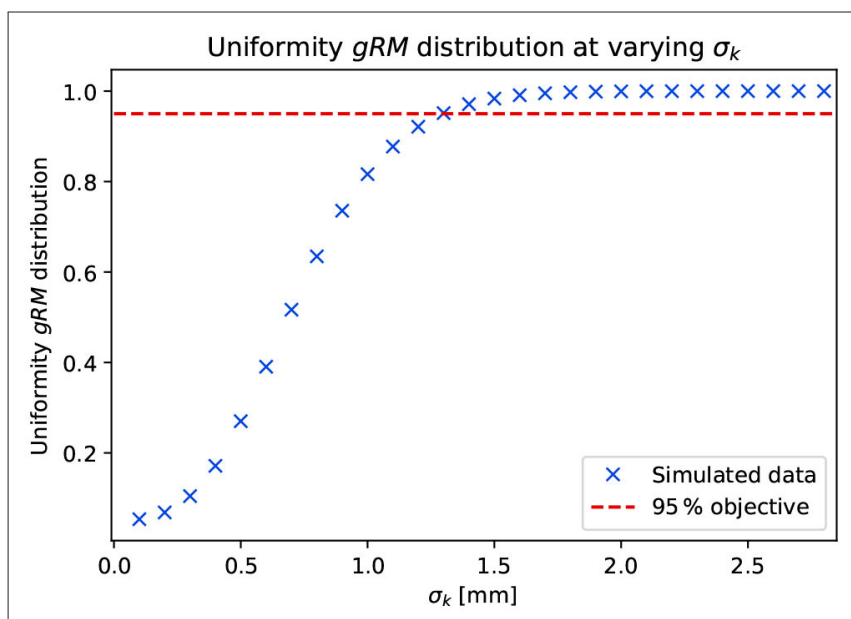

FIGURE 3 | Dependence of the uniformity of the ROS spatial distribution with respect to the value of $\sigma_{k}$ adopted in the convolution of the physical dose distribution. The parameterization of the physical dose distribution is analog to what is presented in Figure 1.
The MRT experiment by Regnard et al. was conducted with synchrotron radiation at the beamline dedicated to biomedical research of the ESRF [19]. Compared to the previously described experiments, MRT at synchrtoron facilities can achieve significantly higher dose rates and therefore shorter irradiation times. The authors reported that the effective beam-on time was adjusted as a function of the ring current. The duration of the irradiation was approximately $1 \mathrm{~s}$, value which we used for our analysis.

It should be noted that the depth at which the MBRT are investigated is a free parameter. The depths were fixed in the previous experimental studies in relation to the tumor location. The model presented in the current study takes in account the dose distribution in the tumor, independently from its depth in the animal. Therefore, the model is applicable at any arbitrary depth, as long as the dose distribution is available.

\section{RESULTS}

\subsection{Calculation of $\sigma_{k}$ in Previous MBRT Studies}

The requirement of 95 to $107 \%$ coverage was respected as soon as $g R M$ reached a lateral distribution with uniformity $>0.95$. Figure 3 shows the evolution of the $g R M$ uniformity with respect to the value assumed by $\sigma_{k}$. A steep increase is observed for approximately $0.5 \mathrm{~mm}<\sigma_{k}<1.5 \mathrm{~mm}$. Outside this range, the shape of the $g R M$ distribution is dominated by the $c-t-c$ value $\left(\sigma_{k}<0.5 \mathrm{~mm}\right)$ or by the convolution kernel $\left(\sigma_{k}>\right.$ $1.5 \mathrm{~mm})$. The $\hat{\sigma}_{k}$ lies within the previously mentioned range. Therefore, its value depends simultaneously on the distribution of the physical dose and on the diffusion properties of $g R M$. Deviations smaller than $3 \%$ were observed when comparing $\hat{\sigma}_{k}$ calculated from Gaussian-shaped mini-beams and rectangularshaped mini-beams. The latter was used in the rest of the study.
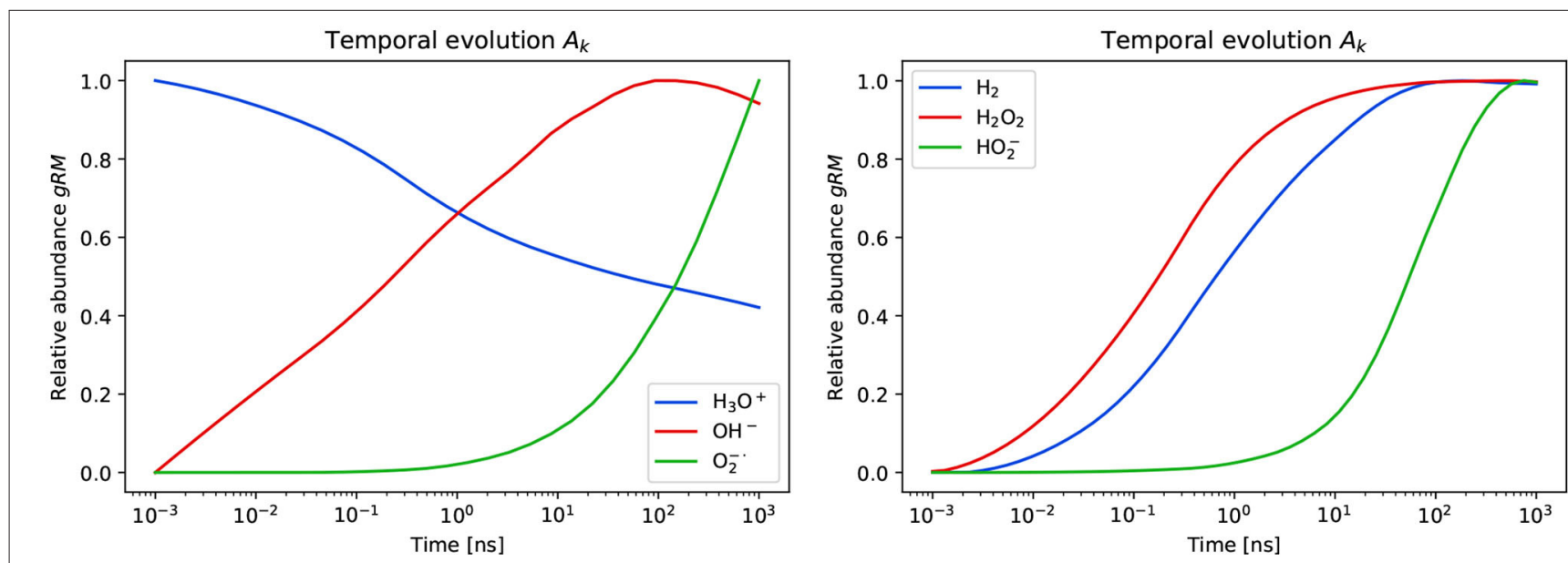

FIGURE 4 | Temporal evolution of the amount of molecules and radicals produced in the TRAX-CHEM simulations. The abundance of $g$ RM are normalized by the maximum values reached within the time range of the simulations. A sub-set of six among the twelve simulated species are shown. These are further sub-divided in the first (left) and second (right) category depending on the behavior at the latest simulation time point. 

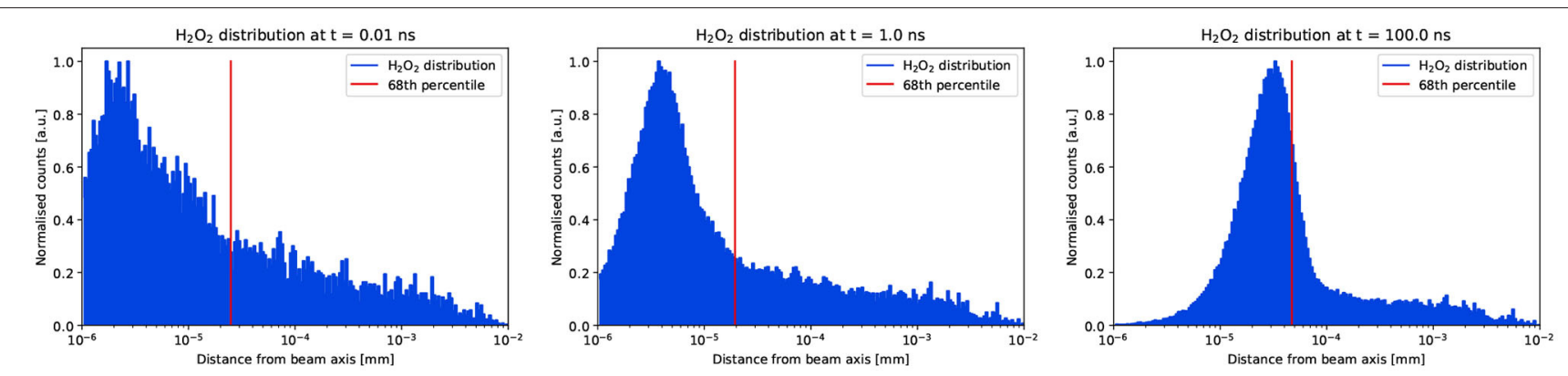

FIGURE 5 | Instantaneous spatial distributions of the hydrogen peroxide sampled at three time points. The blue histograms show the data extracted from the TRAX-CHEM simulations and the red vertical lines show the position of $P_{68}$. The counts are normalized to the maximum at every time point.

The values obtained for $\hat{\sigma}_{k}$ were

$$
\hat{\sigma}_{k}= \begin{cases}1293 \mu \mathrm{m} & \text { for Prezado et al. [8] } \\ 149 \mu \mathrm{m} & \text { for Dombrowsky et al. [6] } \\ 83 \mu \mathrm{m} & \text { for } 200^{*} \text { in Regnard et al. [9] } \\ 41 \mu \mathrm{m} & \text { for 100LR in Regnard et al. [9] }\end{cases}
$$

The calculation of the values (3) did not assume any a priori information regarding the generation and diffusion properties of the chemical species. We observe that the values required by $\hat{\sigma}_{k}$ are approximately half the $c-t-c$ distance.

\subsection{Temporal Evolution of $\boldsymbol{A}_{\boldsymbol{k}}$ and $\sigma_{k}$}

The dependency of $A_{k}$ on the time point of the TRAX-CHEM simulation is presented in Figure 4. The two categories of chemical species are reported in two separate plots. Only a sub-set of the species in the first category is shown for conciseness. The ones excluded from the plots are not relevant for the following steps of the current study. The steady state was reached by the following:

$$
\mathrm{H}_{2} \mathrm{O}_{2}, \mathrm{H}_{2}, \mathrm{HO}_{2}^{-}
$$

while the remaining species listed in Table 1 did not respect $\partial A_{k} / \partial t \simeq 0$. All three in (4) respected the condition (iii). Among them, only $\mathrm{H}_{2} \mathrm{O}_{2}$ respected the condition (iv), which is a reactive oxygen species (ROS) and can be expected to be associated with cellular damage [14]. Therefore, the further analysis is restricted to hydrogen peroxide. For this ROS, the condition $\partial A_{k} / \partial t \simeq 0$ is respected. In particular, for $t>50 \mathrm{~ns}$ we observed deviations of $A_{k}(t)$ smaller than $0.25 \%$ from its mean value. Therefore, we set $t_{1}=50 \mathrm{~ns}$.

The time-dependent histograms representing the spatial distribution of the hydrogen peroxide radicals are shown in Figure 5. The values of $P_{68}$ are calculated and reported in the plot. At positions close to the beam axis, the number of $\mathrm{H}_{2} \mathrm{O}_{2}$ decreases with increasing time due to the diffusion. A minimum is observed in Figure 5 for $t=100$ ns due to the logarithmic representation of the distance from the beam axis and the ordinate scale. This takes into account the total number of molecules at a given radial distance from the beam axis and does not normalize by the volume over which such molecules are

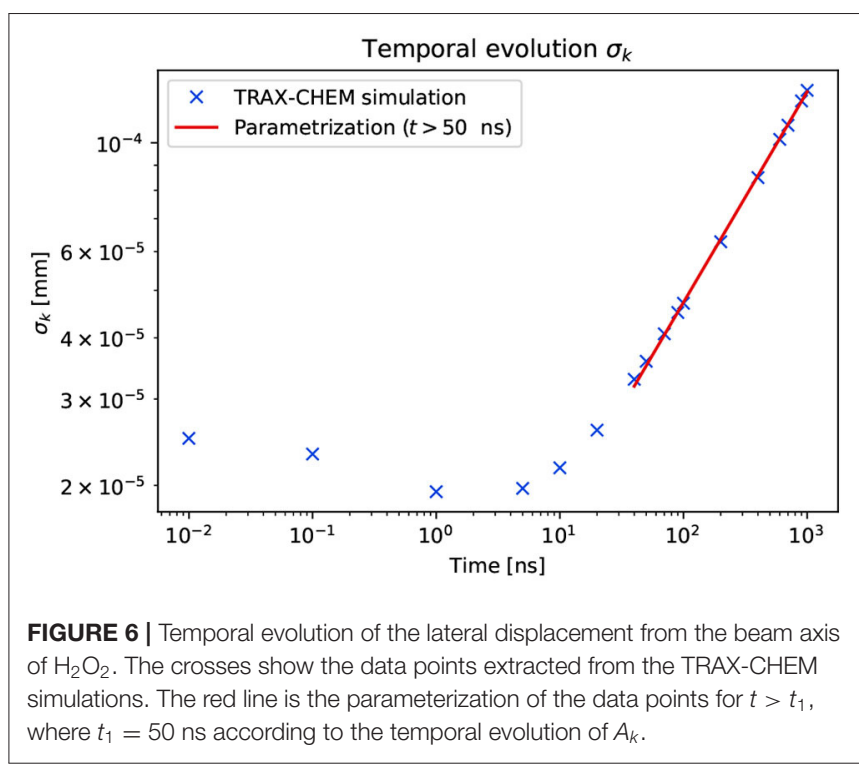

distributed. The same data, plotted with a normalization by the volume is provided in the Supplementary Material of the article. The values $P_{68}$ were then used to parameterize $\sigma_{k}(t)$.

The evolution of $\sigma_{k}(t)$ and the power law fit for $t>t_{1}$ are shown in Figure 6. This was parameterized with the variables in SI units. The fit parameters were:

$$
\left\{\begin{array}{l}
a=(4.8 \pm 0.2) \cdot 10^{-5} \mathrm{~m} \\
b=(4.3 \pm 0.3) \cdot 10^{-1} \\
t_{0}=1 \mathrm{~s}
\end{array}\right.
$$

The parameterization (5) was used to extrapolate $\sigma_{k}(t)$ for times greater than the last simulation time point.

\subsection{Tumor Coverage by $\mathrm{H}_{2} \mathrm{O}_{2}$ in Previous MBRT Animal Experiments}

The minimum widths of the Gaussian kernels to ensure coverage of the target by $\mathrm{H}_{2} \mathrm{O}_{2}$ were reported in Equation (3). Inserting the values (5) into (2), we obtained the following minimum 


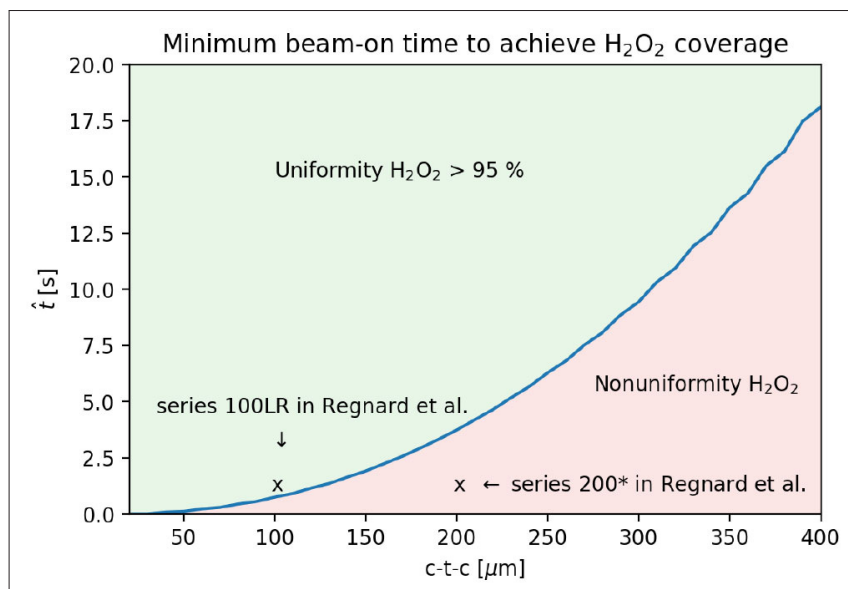

FIGURE 7 | Prediction of the minimum beam-on time required to achieve uniform $\mathrm{H}_{2} \mathrm{O}_{2}$ coverage for syncrotron experiments with $P V D R=71$ and beam width $25 \mu \mathrm{m}$. The prediction is compared with previous experimental data [9].

irradiation times that would ensure the required diffusion of hydrogen peroxide in the previous MBRT animal experiments:

$$
\hat{t}= \begin{cases}2120 \pm 240 \mathrm{~s} & \text { for Prezado et al. [8] } \\ 13.9 \pm 1.5 \mathrm{~s} & \text { for Dombrowsky et al. [6] } \\ 3.5 \pm 0.4 \mathrm{~s} & \text { for } 200^{*} \text { in Regnard et al. [9] } \\ 0.70 \pm 0.08 \mathrm{~s} & \text { for 100LR in Regnard et al. [9] }\end{cases}
$$

while the experimental irradiation times were

$$
t_{\exp }= \begin{cases}2100 \mathrm{~s} & \text { for Prezado et al. [8] } \\ 300 \mathrm{~s} & \text { for Dombrowsky et al. [6] } \\ 1 \mathrm{~s} & \text { for Regnard et al. [9] }\end{cases}
$$

In three out of the four experiments analyzed, it was valid that $t_{\text {exp }} \geq \hat{t}$. In such cases, according to the model presented, the $\mathrm{H}_{2} \mathrm{O}_{2}$ provided a coverage between 95 and $107 \%$ of its mean concentration. As a matter of fact these experiments were associated with high probabilities of tumor ablation or growth delay. For the series $200^{*}$ in Regnard et al., the uniform coverage of $\mathrm{H}_{2} \mathrm{O}_{2}$ was not reached during beam-on, since $t_{\exp }<\hat{t}$. This should be attributed to the high dose rate achievable at a synchrotron and an increased $c-t-c$ spacing. The group $200^{*}$ registered 2 ablated tumors in 32 irradiated rats. The series 100RL produced 5 tumor ablations out of 11 rats.

We further investigated the differences between the series $200^{*}$ and 100RL. The worst case scenario to achieve $\mathrm{H}_{2} \mathrm{O}_{2}$ coverage was taken into account, i.e., the maximum $P V D R=71$ reported at the skin entrance by [9]. We calculated for varying $c-t-c$ what would be the minimum $\hat{t}$ predicted by our model. The result and the comparison with the experimental data is presented in Figure 7. The series 100LR belongs to the region with uniform $\mathrm{H}_{2} \mathrm{O}_{2}$ distribution, while the series $200^{*}$ is attributed to the one where such uniformity is not achieved.

\section{DISCUSSION}

This simulation study identified the distribution of hydrogen peroxide as the candidate to interpret the mini-beam and microbeam efficacy. This molecule is a product of water radiolysis, it is stable and it rapidly reaches the steady state in production versus removal. Moreover, the $\mathrm{H}_{2} \mathrm{O}_{2}$ has a strong oxidizing capacity leading to damage of proteins, lipids, and DNA. Such known properties are now combined with the observation that the distribution of hydrogen peroxide had become uniform in previous MBRT animal experiments where tumor control was achieved. The plausibility of our hypothesis that a radical or molecule can be used as surrogate to describe the biological effect induced by MBRT is therefore confirmed and hydrogen peroxide was identified as the chemical candidate. This is further supported by the different tumor ablation probability reported by Regnard et al. between the group $200^{\star}$ and 100LR. In the former, the uniform $\mathrm{H}_{2} \mathrm{O}_{2}$ coverage was not reached during beam-on and the fraction of animals with tumor ablation was seven-fold smaller compared to the 100LR series, in which $\mathrm{H}_{2} \mathrm{O}_{2}$ diffused uniformly over the target.

The presented model aimed to identify a candidate for the biological efficacy of MBRT in tumors. The investigation of the differential effect between the former and normal tissue was outside the scope of the current study. Nonetheless, the identification of the hydrogen peroxide as the surrogate to minibeam and micro-beam efficacy can be used as a starting point for such analysis. We discuss two phenomena, one physical and one biological, which act in favor of a differential effect between tumor and normal tissue. The first, related to the physics of MBRT, applies especially to pMBRT. We hypothesized that the tumor control is obtained because a homogeneous distribution of hydrogen peroxide covers the target volume. Such coverage is obtained due to a well-tuned combination of proton mini-beam size and center-to-center spacing, which combined with $\mathrm{H}_{2} \mathrm{O}_{2}$ diffusion during beam-on, covers uniformly the tumor. On the other hand, for pMBRT, the PVDR is high at the normal tissue in the entry channel and it degrades with depth until reaching the target [11]. Therefore, while an homogeneous $\mathrm{H}_{2} \mathrm{O}_{2}$ distribution is reached at the tumor, this is not always the case for the normal tissue. In such case, dose volume effects may apply also to the hydrogen peroxide distribution and therefore lead to a higher tolerance of pMBRT in the normal tissue. This applies especially to pMBRT experiments tuning the beam energy to deliver the Bragg peak at the tumor location. The second, related to ROS biology, applies also to MRT. Cancer cell have significantly higher intracellular ROS levels relative to normal healthy cells [23]. For this reason we believe that normal cells have a higher tolerance to ROS relative to cancer cells, making them more resistant to oxidative stress induced by MBRT through $\mathrm{H}_{2} \mathrm{O}_{2}$. Despite these two phenomena, the differential effect in normal and tumor tissue is a key aspect of MBRT and will require further investigation. The following discussion will focus on the biological efficacy of MBRT in tumors.

This work was a simulation study aiming to provide further interpretation of previous animal experiments. Few simplification and assumptions were made. We did not aim to 
reproduce the physical dose distribution irradiated to the animals in the previous studies with the highest accuracy achievable. This was out of the scope of the current work and it is instead the objective of studies dedicated to the beam design [11] or treatment plans evaluation [24]. On the other hand, these simplifications should not affect the results. The chosen approach consisted in defining the physical dose distribution based on the parameters $c-t-c, F W H M$, and PVDR reported by the authors. The simplest model based on rectangular-shaped $D(x)$ was compared to a more realistic $D(x)$ made of multiple Gaussian peaks (Figure 1). The deviation in the estimation of $\hat{\sigma}_{k}$ with the two models was limited to the percent level. Therefore, it can be assumed that a further refinement of the $D(x)$ from the multiple Gaussian peaks to a more complex simulation reproducing with high fidelity the previous MBRT experiments would not contribute significantly to the accuracy of the results presented in this work. Therefore, the use of rectangular-shaped $D(x)$ distributions is justified. The little susceptibility of $\hat{\sigma}_{k}$ to small deviations of $D(x)$ could be interpreted in the light of the data presented in Figure 3. The ICRU objective of $95 \%$ uniformity is reached at the upper end of the steep part of the curve, i.e., toward the part where the curve tends asymptotically to $100 \%$ uniformity. Among the parameters in (1), this part of the curve is mostly dependent on $\sigma_{k}$. Therefore, the spatial and temporal diffusion properties of the hydrogen peroxide provide a greater contribution compared to small deviations in the distribution of the physical dose. The ICRU objective of $95 \%$ was assumed without error in this study. However, this recommendation is the result of a long history of clinical experience in many different conditions and therefore should not be assumed as valid a priori for every application. Since the $95 \%$ of the dose is a minimum objective, it means that lower values may still provide the desired biological endpoint. For what concerns our study, lower uniformity values are correlated to shorter irradiation times required to achieve the desired $g R M$ distribution. Therefore, even in presence of a lower objective such as $90 \%$, the values presented in (6) would still support the applicability of our model when compared to (7). The maximum dose objective of $107 \%$ does not enter directly in our model. Finally it should be reminded that MBRT introduces a change of paradigm. Therefore there is no evidence yet that the parameters defined in the ICRU are strictly required to observe tumor control. This study investigated whether a generalization of such recommendation, which would still be valid and applicable in the conventional therapy, could be adopted in MBRT experiments. The current chemical-stage analysis comes as a complement to previous studies analyzing the biological effects of spatially fractionated dose distributions. While not all the biological phenomena, such as the higher tolerance of normal tissue, could be described through our proposed model; we showed how the spatial distribution of $\mathrm{H}_{2} \mathrm{O}_{2}$ is a potential highly relevant parameter and should be further investigated. The correct interpretation of such microscopic mechanisms of action is required in order for spatially fractionated radiation to advance toward a potential future clinical implementation [25].

One further assumption in the study was the extrapolation of the parameterization $\sigma_{k}(t)$ to larger time scales compared to the ones simulated with TRAX-CHEM. In particular, the simulations ran until $10^{-5} \mathrm{~s}$ while the interpretation of the previous animal experiments required times up to the order of $10^{2} \mathrm{~s}$ for microbeams and $10^{3} \mathrm{~s}$ for mini-beams. This extrapolation is supported by three facts. First, the distribution of $\mathrm{H}_{2} \mathrm{O}_{2}$ is parameterized solely by $A_{k}(t)$ and $\sigma_{k}(t)$ in the current study. The TRAX-CHEM simulations were run until it was verified that $\partial A_{k} / \partial t \simeq 0$ was respected and $\sigma_{k}(t)$ was the only varying quantity. Deviations of $A_{k}(t)$ smaller than $0.25 \%$ were observed from $t_{1}$ until the latest simulation point, i.e., over 3 orders of magnitude in time. Further simulations beyond this time point would require additional computational burden without a significant increase of the results accuracy. Second, the physical properties of hydrogen peroxide are close to the ones of water [26]. For example, its dipole moment is only $22 \%$ larger than the one of $\mathrm{H}_{2} \mathrm{O}$, it is uncharged and protonated at physiological $\mathrm{pH}$. Therefore, the diffusion in the extracellular space of hydrogen peroxide is not expected to be dumped and it can reach extended spatial distances. Moreover, hydrogen peroxide is commonly treated as a molecule that freely crosses membranes, with only some exceptions [26]. Such property, combined with the condition (iv), supports the choice of $\mathrm{H}_{2} \mathrm{O}_{2}$ as a surrogate for radiation damage to the cells. Third, this extrapolation was based on the property (i), which is the long term stability of the hydrogen peroxide. This was simulated by TRAX-CHEM in water. Shorter times can be expected in a cellular environment, where the hydrogen peroxide is removed by specific enzymes [27]. Nonetheless, the stability of $\mathrm{H}_{2} \mathrm{O}_{2}$ concentration over several minutes up to the hour is observed also in cell cultures [28]. The stability of the concentration investigated in the current work is limited to the beam-on time. Therefore, the extrapolation of the data presented in Figure 6 to larger time points is justified. Future work may include investigations with more realistic phantoms for the simulations and a comparison to biological data. Moreover, the absolute concentration of $\mathrm{H}_{2} \mathrm{O}_{2}$ should also be the subject of investigation of future more advanced simulations taking into account the surrounding biological environment. A comparison of the ROS levels in the experiments and the simulation simulation predictions can then be performed. In the current study we adopted a simple geometry in order to investigate the basic phenomena related to out hypothesis. Finally, the comparison between different simulation codes such as TOPAS-nBio [29], [30], and PARTRAC [31] may also be of interest in future stages of the project.

The work presented supports the potential explanation of the MBRT anti-tumor efficacy though the distribution of $\mathrm{H}_{2} \mathrm{O}_{2}$. The current study does not demonstrate the correlation between the hydrogen peroxide concentration and the tumor control. Further experimental studies will be necessary to assess such correlation. If this is confirmed, the concentration of this ROS can be used as a marker for the MBRT effect. Ideally, the level of hydrogen peroxide will have to be measured by dedicated experiments. The predictions obtained with the simulations should be confirmed with a direct measurement of the absolute $\mathrm{H}_{2} \mathrm{O}_{2}$ concentration in real-time during beam-on with dedicated reagents, e.g., Amplex ${ }^{\mathrm{TM}}$ Red [32]. This would allow not only to experimentally benchmark the kernel used for the conversion from physical 
dose to ROS concentration, but also to assess the absolute value of $\rho_{g R M}(x)$ in the experimental setup. The level of $\mathrm{H}_{2} \mathrm{O}_{2}$ after the diffusion process should then be correlated to the tumor control parameters in animal experiments. Damaging reactions caused by ROS are known to induce cell death by oxidative stress. Previous studies show that even at concentrations below the required threshold, single ROS activated protein can lead to cell death [33]. Moreover, $\mathrm{H}_{2} \mathrm{O}_{2}$ is involved in developmental control by triggering apoptosis and cell proliferation [34]. Finally, an additional model describing the differential response of normal tissue and tumor would be required to interpret the absence of brain damage observed in the proton mini-beam experiments [8]. The investigation of additional models, the specific mechanisms involved and the correlation between the hydrogen peroxide concentration and the tumor control are left for future studies. According to the results presented in this work, we limit the discussion in presenting $\mathrm{H}_{2} \mathrm{O}_{2}$ as the candidate to interpret the mini-beam and micro-beam efficacy.

\section{CONCLUSION}

A simulation study to investigate a potential surrogate to describe the efficacy of mini-beam and micro-beam radiation therapy was presented. The hydrogen peroxide fulfilled the required conditions; namely, it is a product of water radiolysis, it is stable, it reaches rapidly the steady state in production versus removal and it has oxidizing capacity to allow damage of proteins, lipids, or DNA. It was demonstrated that the $\mathrm{H}_{2} \mathrm{O}_{2}$ produced in the dose-peaks diffuses to the dose-valleys during beam-on leading to a homogeneous ROS distribution over the target. This distribution respected the reinterpretation of the ICRU recommendation for target coverage, while this was not respected by the physical dose. The model was tested on three previous independent photon micro-beam and proton mini-beam animal experiments. When the predicted minimum irradiation time matched the experimental data, the biological

\section{REFERENCES}

1. Citrin DE. Recent developments in radiotherapy. New Engl J Med. (2017) 377:1065-75. doi: 10.1056/NEJMra1608986

2. Teoh M, Clark CH, Wood K, Whitaker S, Nisbet A. Volumetric modulated arc therapy: a review of current literature and clinical use in practice. Br J Radiol. (2011) 84:967-96. doi: 10.1259/bjr/22373346

3. Durante M, Orecchia R, Loeffler JS. Charged-particle therapy in cancer: clinical uses and future perspectives. Nat Rev Clin Oncol. (2017) 14:483-95. doi: 10.1038/nrclinonc.2017.30

4. Durante M, Golubev A, Park WY, Trautmann C. Applied nuclear physics at the new high-energy particle accelerator facilities. Phys Rep. (2019) 800:1-37. doi: 10.1016/j.physrep.2019.01.004

5. Bräuer-Krisch E, Serduc R, Siegbahn EA, Le Duc G, Prezado Y, Bravin A, et al. Effects of pulsed, spatially fractionated, microscopic synchrotron X-ray beams on normal and tumoral brain tissue. Mutat Res. (2010) 704:160-6. doi: 10.1016/j.mrrev.2009.12.003

6. Dombrowsky AC, Burger K, Porth AK, Stein M, Dierolf M, Günther B, et al. A proof of principle experiment for microbeam radiation therapy at the Munich compact light source. Radiat Environ Biophys. (2019) 59:111-20. doi: 10.1007/s00411-019-00816-y effect was maximum. A reduced tumor ablation probability was observed in one experiment in which the $\mathrm{H}_{2} \mathrm{O}_{2}$ did not cover uniformly the target. We can conclude that the hydrogen peroxide is a good candidate to describe the mini-beam and micro-beam efficacy.

\section{DATA AVAILABILITY STATEMENT}

The raw data supporting the conclusions of this article will be made available by the authors, without undue reservation.

\section{AUTHOR CONTRIBUTIONS}

JS proposed the concept of $\mathrm{H}_{2} \mathrm{O}_{2}$ as a surrogate and designed the objectives and structure of the study. $\mathrm{RD}$ planned the investigation strategy and conducted the study. TB defined the input parameters, ran the simulations, and exported the data to generate the plots included in the manuscript. MF and MK provided access to the TRAX-CHEM simulation code and tuned it to the objectives of the current study. All the authors contributed to the interpretation of the results. All authors read and approved the final manuscript.

\section{ACKNOWLEDGMENTS}

RD was supported by the International Max Planck Research School for Quantum Dynamics in Physics, Chemistry and Biology, Heidelberg, Germany. The authors thank the three reviewers for the constructive feedback during the review process.

\section{SUPPLEMENTARY MATERIAL}

The Supplementary Material for this article can be found online at: https://www.frontiersin.org/articles/10.3389/fphy. 2020.564836/full\#supplementary-material

7. Prezado Y, Jouvion G, Guardiola C, Gonzalez W, Juchaux M, Bergs J, et al. Tumor control in RG2 glioma-bearing rats: a comparison between proton minibeam therapy and standard proton therapy. Int J Radiat Oncol Biol Phys. (2019) 104:266-71. doi: 10.1016/j.ijrobp.2019.01.080

8. Prezado Y, Jouvion G, Patriarca A, Nauraye C, Guardiola C, Juchaux M, et al. Proton minibeam radiation therapy widens the therapeutic index for high-grade gliomas. Sci Rep. (2018) 12:16479. doi: 10.1038/s41598-01834796-8

9. Regnard P, Duc GL, Bräuer-Krisch E, Troprés I, Siegbahn EA, Kusak A, et al. Irradiation of intracerebral 9L gliosarcoma by a single array of microplanar $\mathrm{x}$ ray beams from a synchrotron: balance between curing and sparing. Phys Med Biol. (2008) 53:861-78. doi: 10.1088/0031-9155/53/4/003

10. Guardiola C, Peucelle C, Prezado Y. Optimization of the mechanical collimation for minibeam generation in proton minibeam radiation therapy. Med Phys. (2017) 44:1470-8. doi: 10.1002/mp.12131

11. Schneider T, De Marzi L, Patriarca A, Prezado Y. Advancing proton minibeam radiation therapy: magnetically focussed proton minibeams at a clinical centre. Sci Rep. (2020) 10:1-10. doi: 10.1038/s41598-020-58052-0

12. Dilmanian FA. Response of rat intracranial 9L gliosarcoma to microbeam radiation therapy. Neuro-Oncology. (2002) 4:26-38. doi: $10.1215 / 15228517-4-1-26$ 
13. Dilmanian FA, Qu Y, Feinendegen LE, Pe na LA, Bacarian T, Henn FA, et al. Tissue-sparing effect of x-ray microplanar beams particularly in the CNS: is a bystander effect involved? Exp Hematol. (2007) 35(4 Suppl.):69-77. doi: 10.1016/j.exphem.2007.01.014

14. Montay-Gruel P, Acharya MM, Petersson K, Alikhani L, Yakkala C, Allen $\mathrm{BD}$, et al. Long-term neurocognitive benefits of FLASH radiotherapy driven by reduced reactive oxygen species. Proc Natl Acad Sci USA. (2019) 166:10943-51. doi: 10.1073/pnas.1901777116

15. Krämer $M$, Durante $M$. Ion beam transport calculations and treatment plans in particle therapy. Eur Phys J D. (2010) 60:195-202. doi: 10.1140/epjd/e2010-00077-8

16. Sies H, Berndt C, Jones DP. Oxidative stress. Annu Rev Biochem. (2017) 86:715-48. doi: 10.1146/annurev-biochem-061516-045037

17. Boscolo D, Krämer M, Durante M, Fuss MC, Scifoni E. TRAX-CHEM: a pre-chemical and chemical stage extension of the particle track structure code TRAX in water targets. Chem Phys Lett. (2018) 698:11-8. doi: 10.1016/j.cplett.2018.02.051

18. Landberg T, Chavaudra J, Dobbs J, Gerard JP, Hanks G, Horiot JC, et al. Report 62. J Int Commiss Radiat Units Measure. (1999) 32:NP. doi: 10.1093/jicru/os32.1.Report62

19. Elleaume H, Charvet AM, Berkvens P, Berruyer G, Brochard T, Dabin $\mathrm{Y}$, et al. Instrumentation of the ESRF medical imaging facility. Nuclear Instrum Methods Phys Res. (1999) 428:513-527. doi: 10.1016/S0168-9002(99) 00167-9

20. Krämer M, Kraft G. Calculations of heavy-ion track structure. Radiat Environ Biophys. (1994) 33:91-109. doi: 10.1007/BF01219334

21. Boscolo D, Krämer M, Fuss MC, Durante M, Scifoni E. Impact of target oxygenation on the chemical track evolution of ion and electron radiation. Int J Mol Sci. (2020) 21:424. doi: 10.3390/ijms21020424

22. Zaider M, Vracko MG, Fung AYC, Fry JL. Electron transport in condensed water. Proc Eleventh Sympos Microdosim. (1994) 52:139. doi: $10.1093 / \mathrm{rpd} / 52.1-4.139$

23. Perillo B, Di Donato M, Pezone A, Di Zazzo E, Giovannelli P, Galasso G, et al. ROS in cancer therapy: the bright side of the moon. Exp Mol Med. (2020) 52:192-203. doi: 10.1038/s12276-020-0384-2

24. Lansonneur P, Mammar H, Nauraye C, Patriarca A, Hierso E, Dendale R, et al. First proton minibeam radiation therapy treatment plan evaluation. Sci Rep. (2020) 10:7025. doi: 10.1038/s41598-02063975-9

25. Coleman $\mathrm{CN}$, Ahmed MM. Implementation of new biology-based radiation therapy technology: when is it ready so "perfect makes practice?" Int J Radiat Oncol Biol Phys. (2019) 105:934-7. doi: 10.1016/j.ijrobp.2019. 08.013
26. Bienert GP, Schjoerring JK, Jahn TP. Membrane transport of hydrogen peroxide. Biochim Biophys Acta. (2006) 1758:994-1003. doi: 10.1016/j.bbamem.2006.02.015

27. Ng CF, Schafer FQ, Buettner GR, Rodgers VGJ. The rate of cellular hydrogen peroxide removal shows dependency on GSH: mathematical insight into in vivo $\mathrm{H} 2 \mathrm{O} 2$ and GPx concentrations. Free Radic Res. (2007) 41:1201-11. doi: 10.1080/10715760701625075

28. Kern M, Fridrich D, Reichert J, Skrbek S, Nussher A, Hofem S, et al. Limited stability in cell culture medium and hydrogen peroxide formation affect the growth inhibitory properties of delphinidin and its degradation product gallic acid. Mol Nutr Food Res. (2007) 51:1163-72. doi: 10.1002/mnfr.200700004

29. Schuemann J, McNamara AL, Ramos-Méndez J, Perl J, Held KD, Paganetti $\mathrm{H}$, et al. TOPAS-nBio: an extension to the TOPAS simulation toolkit for cellular and sub-cellular radiobiology. Radiat Res. (2018) 191:125. doi: $10.1667 / R R 15226.1$

30. Incerti S, Douglass M, Penfold S, Guatelli S, Bezak E. Review of Geant4DNA applications for micro and nanoscale simulations. Phys Med. (2016) 32:1187-200. doi: 10.1016/j.ejmp.2016.09.007

31. Friedland W, Dingfelder M, Kundrát P, Jacob P. Track structures, DNA targets and radiation effects in the biophysical Monte Carlo simulation code PARTRAC. Mutat Res. (2011) 711:28-40. doi: 10.1016/j.mrfmmm.2011.01.003

32. Victorelli S, Passos JF. Reactive oxygen species detection in senescent cells. In: Methods in Molecular Biology. Vol. 1896. New York, NY: Humana Press Inc. (2019). p. 21-9. doi: 10.1007/978-1-4939-8931-7_3

33. Wagner D, Przybyla D, Op Den Camp R, Kim C, Landgraf F, Keun PL, et al. The genetic basis of singlet oxygen-induced stress response of Arabidopsis thaliana. Science. (2004) 306:1183-5. doi: 10.1126/science.1103178

34. Hachiya M, Akashi M. Catalase regulates cell growth in HL60 human promyelocytic cells: evidence for growth regulation by $\mathrm{H} 2 \mathrm{O} 2$. Radiat Res. (2005) 163:271-82. doi: 10.1667/RR3306

Conflict of Interest: The authors declare that the research was conducted in the absence of any commercial or financial relationships that could be construed as a potential conflict of interest.

Copyright (C) 2020 Dal Bello, Becher, Fuss, Krämer and Seco. This is an open-access article distributed under the terms of the Creative Commons Attribution License (CC $B Y)$. The use, distribution or reproduction in other forums is permitted, provided the original author(s) and the copyright owner(s) are credited and that the original publication in this journal is cited, in accordance with accepted academic practice. No use, distribution or reproduction is permitted which does not comply with these terms. 